\title{
Particle acceleration in Blazars
}

\author{
Matthias Weidinger ${ }^{1}$ and Felix Spanier ${ }^{2}$ \\ Lehrstuhl für Astronomie, Universität Würzburg, \\ Am Hubland, 97074 Würzburg, Germany \\ ${ }^{1}$ email: mweidinger@astro.uni-wuerzburg.de \\ ${ }^{2}$ email: fspanier@astro.uni-wuerzburg.de
}

\begin{abstract}
Understanding the variable emission of blazars observed with gamma-ray telescopes and Fermi has become a major challenge for theoretical models of particle acceleration. Here, we introduce a novel time-dependent emission model in which the maximum energy of particles is determined from a balance between Fermi type I and II acceleration energy gains and radiative energy losses, allowing for an explanation of both the characteristic spectral energy distribution of blazars and their intrinsic sub-hour variability. Additionally, we can determine the physical condition of the emitting plasma concerning its turbulence and typical shock speeds.
\end{abstract}

Keywords. acceleration of particles, shock waves, radiation mechanisms: nonthermal, galaxies: active, galaxies: jets, BL Lacertae objects: individual (1 ES 1218+30.4, PKS 2155-304)

\section{Introduction}

Blazars, with all their subcategories from Flat Spectrum Radio Quasars (FSRQs) to Low-, Intermediate- and High Frequency Peaked BL-Lac Objects (LBLs, IBLs, HBLs), are believed to be Active Galactic Nuclei (AGN) where the highly relativistic, collimated outflow from the central black hole emerges under a small angle to the line of sight (Urry \& Padovani 1995). The blazar flavours can be distinguished based on their spectral features. While all types show a more or less pronounced double humped spectral energy distribution (SED), FSRQs are the most luminous ones with the first peak occurring arround $10^{14} \mathrm{~Hz}$, in LBLs and IBLs the total luminosity decreases while the peak frequency increases up to $10^{18} \mathrm{~Hz}$ when considering HBLs (e.g. Fossati et al. 1998). The jet as the origin of the characteristic very high energy (VHE) emission from blazars is beyond doubt, but the microphysical processes, the composition as well as typical physical conditions such as the size of the emitting region in the jet are still a matter of debate. To derive those features one has to understand the particle distributions leading to the observed SED in a microphysical way i.e. acceleration mechanisms will be essential. This extends models for the emission processes (e.g. Ghisellini 1988, Chiang \& Böttcher 2002 or Mannheim 1993) where ad-hoc particle spectra are assumed. Fortunately blazars are non-static objects showing variability in their VHE emission down to timescales of minutes, hence providing information about acceleration and cooling processes. Secondly, if all types of blazars are essentially the same objects, the distinctions in their SEDs should as well arise from processes within the jet, this could mean variations in the acceleration mechanism itself or the dominant particle species.

With our "Code On Jetsystems Of Non-thermally Emitting Sources", a box-model considering acceleration due to diffusive shock acceleration as well as all the relevant radiation mechanisms self-consistently and time-dependently, we investigate those properties by comparing model SEDs and lightcurves during outbursts of blazars with multiwavelength observations provided by X-Ray satellites, Fermi-LAT and Air-Cerenkov telescopes. The parameters of the modelling can then be used to find boundaries for 
the properties of the underlying plasma. We apply our model on the low-state emission and variability data of the two HBLs PKS 2155-304 and 1 ES 1218+30.4. The model gives predictions how these objects behave spectrally resolved during a flare, even though only one energy band has been observationally covered and gives hints for their plasmaphysical properties.

\section{The Model}

Here we will give an overview of the main features of the model, for a complete description see Weidinger et al. (2010) and Weidinger \& Spanier (2010b). Our model slightly follows the ansatz of Kirk et al. (1998) and extends it with stochastic acceleration. We solve the corresponding one dimensional Vlasov-equation in the diffusion approximation (see e.g. Schlickeiser 2002) for every particle species $i$ considered in two spatially different zones. A setup is used where the upstream acceleration zone is nested within a bigger radiation zone. Both are assumed to be spherical symmetrical (radius $R_{z}$ ), containing isotropic particle distributions. The kinetic equation in the acceleration zone is

$$
\partial_{t} n_{i}=\partial_{\gamma}\left[\left(\beta_{s, i} \gamma^{2}-t_{\mathrm{acc}, \mathrm{i}}^{-1} \gamma\right) \cdot n_{i}\right]+\partial_{\gamma}\left[\left[(a+2) t_{\mathrm{acc}, \mathrm{i}}\right]^{-1} \gamma^{2} \partial_{\gamma} n_{i}\right]+Q_{0, i}-\frac{n_{i}}{t_{\mathrm{esc}, \mathrm{i}}}
$$

with $Q_{0}$ being a monoenergetic injection function for particles streaming into the considered region of the jet and $\beta_{s}$ the corresponding synchrotron loss rate, $t_{\mathrm{acc}}$ and $a$ can be derived from microphysics to

$$
a=\frac{9}{4} \frac{v_{s}^{2}}{v_{A}^{2}}, \quad t_{\mathrm{acc}}^{-1}=\frac{v_{s}^{2}}{4 K_{\|}}+2 \frac{v_{A}^{2}}{9 K_{\|}}
$$

for a parallel shock and Alfvén waves, here we made use of the hard-sphere approximation (e.g. Lerche \& Schlickeiser 1985). $K_{\|}$is the spatial diffusion coefficient, which for relativistic particles can be estimated as $K_{\|}=1 / 3 c l_{i}$ (Schlickeiser 2002) $\left(l_{i}\right.$ : mean free path). In the steady state this results in a power law, as expected from shock theory. After $t_{\mathrm{esc}, \mathrm{i}}=\eta R_{\mathrm{acc}} / c$ particles enter the radiation zone

$$
\partial_{t} N_{i}=\partial_{\gamma}\left[\left(\beta_{s, i} \gamma^{2}+P_{\mathrm{IC}}(\gamma)\right) \cdot N_{i}\right]-\frac{N_{i}}{t_{\mathrm{rad}, \mathrm{esc}}}+b^{3} \frac{n_{i}}{t_{\mathrm{esc}}}
$$

with $b=R_{\text {acc }} / R_{\text {rad }}$. The IC loss rate is calculated exploiting the full Klein-Nishina-Cross section (e.g. Rüger et al. 2010). Shifting the frame of reference to the Laboratory frame one finds the model SED from the arising photon distribution in the considered region.

\section{Results and Conclusion}

While FSRQs often require different particle species to be modelled, almost every HBL is reproduced using only electrons; i.e. our model is similar to a SynchrotronSelf Compton ansatz, but self-consistent and time-dependent. This model has been applied to the two nearby HBLs 1 ES 1218+30.4 and PKS 2155-304 (Weidinger \& Spanier 2010a, Weidinger \& Spanier 2010b). For both HBLs there is plenty of multiwavelength as well as archival data available. Both show variability in their VHE emission, though PKS 2155-304 with its remarkably short timescales is the more extreme one.

In the first step the low-state SED of an object is reproduced, which then is used to model the lightcurve of the considered flare. Here only the relevant results for the investigation at hand are shown, the complete results and implications are to be found in the individual publications. Fig. 1 shows the SEDs as well as the light curves in different 


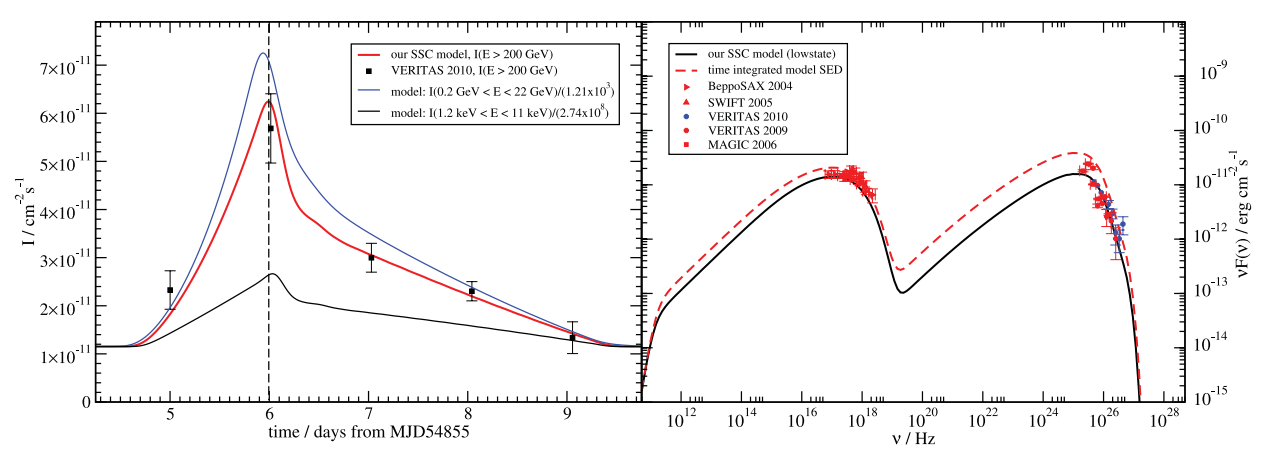

Figure 1. (from Weidinger \& Spanier (2010a)) a) Model lightcurves in different energy bands and the VERITAS measurement from 2009 for 1 ES 1218+30.4. The flare is modeled injecting more $e^{-}$into the steady state emission region for a certain amount of time. b) Low-state model $\mathrm{SED}$, the high-state (dashed line) is computed by averaging over the whole flare shown in a).

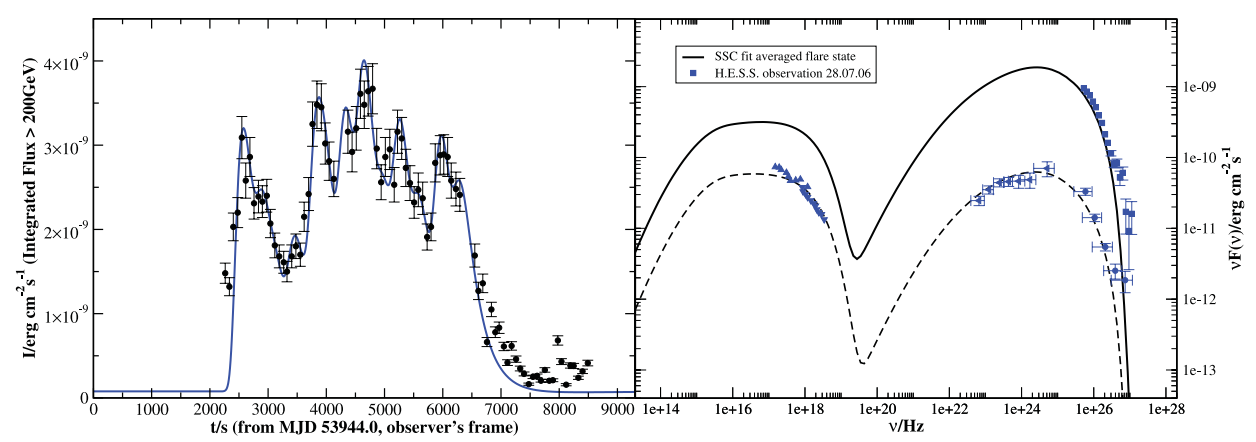

Figure 2. (from Weidinger \& Spanier (2010b)) a) Lightcurve of the famous outburst of PKS 2155-304 measured by H.E.S.S. in 2006 and our model. b) The low-state model SED (dashed line) along with the multiwavelength data of the 2009 campaign with Fermi, this line also matches the archival data of H.E.S.S. from 2003 (Weidinger et al. 2010). The high-state reproducing the H.E.S.S. data is the time average over the whole outburst shown in a).

energy bands for the modelling process described above for all the available data from BeppoSAX, SWIFT, VERITAS and MAGIC (Donato et al. 2005, Tramacere et al. 2007, The VERITAS collaboration 2010, Albert et al. 2006) of 1 ES 1218+30.4 . Although the data is not simultaneous, it can be used for the modelling, for 1 ES $1218+30.4$ is in a low-state most of the time, as the comparison of the VHE data yields. As one can see in Fig. 1a) we are able to explain the outburst recorded by VERITAS with our acceleration model. In Fig. 2 we show the results of applying our model to PKS 2155-304 and its famous outburst. To derive the low-state as a basis for the outburst we used the latest multiwavelength data of Fermi from 2009 (Aharonian et al. 2009). For the flare, density fluctuations as the blob travels down the jet axis, i.e. a varying injection function in eq. (2.1), was assumed. This reproduces the H.E.S.S. data of the event (Aharonian et al. 2007) and proofs the acceleration assumptions to be correct, hence the fit parameters can be used for further investigations (see Table 1).

We model the acceleration process self-consistently, hence we are able to compute upper boundaries for the parameters of the underlying plasma (Table 1 ). Since $l_{i} \ll R_{\text {acc }}$ to ensure the diffusion approximation to remain valid one finds an upper boundary for $K_{\|}$setting $l=R_{\text {acc }}$. For the values of $t_{\text {acc }}$ and $a$ from the modelling of a blazar, this results in an upper boundary for $v_{s}$. In the non-relativistic case this can be used for further investigations because then the scaling for the acceleration-timescale is known 
Table 1. Important parameters used to model the low-state of the two HBLs shown in Fig. 1 and Fig. 2 and the resulting plasma-physical boundaries for the assumed acceleration process using $l=R_{\text {acc }}$.

\begin{tabular}{|l|cccc|ccc|}
\hline & $R_{\mathbf{a c c}}(\mathbf{c m})$ & $t_{\text {acc }}(\mathbf{s})$ & $\mathbf{a}$ & $\alpha^{1}$ & $K_{\| \mid}^{\text {max }}(\mathbf{c m})$ & $v_{s}^{\text {max }}\left(\mathbf{c m ~ s}^{-1}\right)$ & $v_{A}^{\text {max }}\left(\mathbf{c m ~ s}^{-1}\right) \mid$ \\
\hline $1 \mathrm{ES} 1218$ & $6 \cdot 10^{14}$ & $2.22 \cdot 10^{5}$ & 10 & 1.9 & $6 \cdot 10^{24}$ & $9.9 \cdot 10^{9}$ & $4.7 \cdot 10^{9}$ \\
\hline PKS2155 & $1 \cdot 10^{13}$ & $3.77 \cdot 10^{3}$ & 20 & 2.0 & $1 \cdot 10^{23}$ & $1.0 \cdot 10^{10}$ & $3.4 \cdot 10^{10}$ \\
\hline
\end{tabular}

${ }^{1}$ Powerlaw-index of the underlying accelerated electrons.

analytically, eq. (2.2). In highly relativistic cases one might use e.g. PIC simulations (e.g. Spitkovski 2008) for a deeper analysis of the correlation of the acceleration timescale with the energies involved. Nevertheless this gives us a first idea about shock speeds in jets and whether our assumptions were correct. As one can see from Table 1 even for such a large $K_{\|}$the shock speed only reaches up to $0.3 c$. For more realistic values for the spatial diffusion coefficient $\left(\ll 0.01 K_{\|}^{\max }\right)$ the shock seems to be non-relativistic. For the compression ratio we find $r=3.7$ for both considered HBLs as one would expect for a strong non-relativistic shock (e.g. Schlickeiser 2002). Note that $r$ is not to be calculated simply from $\alpha$ since Fermi-II processes together with the synchrotron cooling slightly hardens the spectrum. The $v_{A}$ in Table 1 is calculated from $v_{s}$ and $a$ which directly comes out of our modelling process. In further analysis one might use e.g. MHD simulations to find typical $v_{A}$ which then gives $v_{s}$ (or at least a lower boundary) directly and as long as $v_{s} \ll c$ the diffusion coefficient as well as the compression ratio.

With our model we are able to reproduce the emission of different types of blazars due to different particle species being accelerated within an emitting region along the jet axis. This allows us to derive certain values for the diffusion coefficient and the speed of the accelerating shock, which for HBLs seems to be non-relativistic. This is in agreement with the compression ratio of $r=3.7$ as calculated from the particle spectra. A systematic modelling along the blazar sequence should result in distinctive statements about the main distinctions in those objects.

\section{References}

Aharonian et al. 2007, The Astrophysical Journal Letters, 664 L71

Aharonian et al. 2009, The Astrophysical Journal Letters, 696, L150

Albert et al. 2010, The Astrophysical Journal Letters, 642, L119

Chiang, J. \& Böttcher, M. 2002, The Astrophysical Journal, 564, 92

Donato, D. et al. 2005, Astronomy \&3 Astrophysics, 433, 1163

Fossati, G. et al. 1998, Bulletin of the American Astronomical Society, 30, 768

Ghisellini, G. 1988, Advances in Space Research, 8, 579

Kirk et al. 1998, Astronomy \& Astrophysics, 333, 452

Lerche, I. \& Schlickeiser, R. 1985, Astronomy \& Astrophysics, 151, 408

Mannheim, K. 1993, Astronomy \& Astrophysics, 269, 67

Rüger, M., Spanier, F. \& Mannheim, K. 2010, Mon. Not. R. Astron. Soc., 401, 973

Schlickeiser, R. 2002, Cosmic Ray Astrophysics, Springer, Berlin

Spitkovsky, A. 2008, The Astrophysical Journal Letters, 682, L5

The VERITAS collaboration 2010, The Astrophysical Journal Letters, 709, L163

Tramacere, A. et al. 2007, Astronomy \& Astrophysics, 467, 501

Urry, C. M. \& Padovani, P. 1995, Publ. Astron. Soc. Pac., 107, 803

Weidinger, M., Rüger, M. \& Spanier, F. 2010, Astrophysics and Space Sciences Trans., 6, 1

Weidinger, M \& Spanier, F. 2010a, Astronomy \& Astrophysics, 515, A18

Weidinger, M. \& Spanier, F. 2010b, Int. J. Mod. Phys. D, 19, 887 